\title{
Multi-branched Benzylidene Ketone based Photoinitiators for Multiphoton Fabrication
}

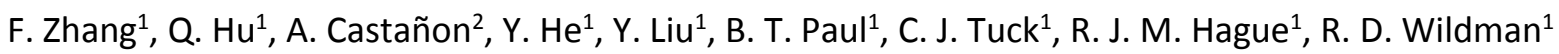 \\ Faculty of Engineering, University of Nottingham, University Park, Nottingham NG2 7RD, United \\ Kingdom
}

Department of Chemistry, University of Nottingham, University Park, Nottingham NG2 7RD, United Kingdom

ABSTRACT: In this article, we report the synthesis of a series of multi-branched benzylidene (BI) ketone-based photo-initiators for two-photon polymerisation based 3D printing/additive manufacturing. Resins prepared by the addition of $1 \mathrm{wt} . \%$ of these initiators were processed in a commercial 2 photon polymerisation system to fabricate 3D woodpile structures, the qualities of which were examined to determine the efficiencies of the initiators. The results showed that compared to commercial initiator Irgacure 369, the four-branched initiator 4-BI exhibited excellent performance with higher writing speeds and broader ideal processing windows. The successful fabrication of complex 3D structures at high writing speeds (up to $100 \mathrm{~K} \mu \mathrm{m} / \mathrm{s}$ ) indicated that the four-branched initiator 4-BI could potentially increase the fabrication efficiency and hence become a promising initiator for two-photon polymerisation.

\section{INTRODUCTION}

Since its inception, two-photon induced photo-polymerisation (TPIP) has been developed towards a manufacturing process that can produce additively manufactured (or 3D printed) structures on nano length scales ${ }^{1}$. This is attractive $-3 D$ printing or additive manufacturing (AM) is a manufacturing method that seeks to avoid traditional manufacturing techniques that are either subtractive (i.e. machining and ablation) or formative (i.e. moulding and casting), and in doing so leverages considerable benefits in terms of design freedom ${ }^{2,3,4,5,6}$. To date, AM is able to fabricate on the super-micron scale ${ }^{3,7}$, but there are significant benefits to also being able to tailor function on the nanoscale, e.g., being able to manufacture on lengths scales that are important for cellular function, or for interacting with the visible part of the electromagnetic spectrum ${ }^{8,9}$. TPIP achieves this level of resolution by employing a two-photon absorption (TPA) procedure, a non-linear process requiring two photons to breach the energy gap between an allowed excited state and the ground state ${ }^{10}$. These non-linearity results in a threshold intensity that induces a photochemical reaction and this can be tailored spatially through the sculpting of the incident beam profile to achieve nanoscale fabrication ${ }^{11}$. For those aiming to manufacture, this conveys advantages of excellent spatial control with high resolution in the sub-micrometer range ${ }^{12,13}$. Moreover, compared to UV light, the long wavelength excitation source $(\sim 780 \mathrm{~nm})$ enables deeper penetration into the resin ${ }^{14}$ as well as a reduction in the number of side reactions ${ }^{1,11}$. These advantages have resulted in TPIP being exploited in a wide range of applications, including three-dimensional (3D) microfabrication ${ }^{15}$, biological 
imaging ${ }^{16}$, the controlled release of biologically relevant species ${ }^{17}$, and high-density optical data storage $^{18}$.

An efficient TPIP process requires a resin containing a highly active two-photon absorption photoinitiator (TPA PI) to achieve a high writing speed and low polymerisation threshold that a high quality structure requires ${ }^{1,19,20,21}$. The most commonly available PIs have been those produced to work with UV illumination, but these have significant drawbacks, notably including low two-photon cross-sections $\left(\sigma_{T P A}\right)^{22}$, high excitation power and long exposure time, all of which combine to result in poor quality structures ${ }^{1}$. In recent years, there has been a shift to the production of dipolar or quadrupolar TPA chromophores containing strong electron donors and/or acceptors, as well as planar $\pi$ systems with long conjugation length ${ }^{1,11,12,23,24}$. These have, however, been slow to achieve widespread use ${ }^{1}$.

One recent development has been the production of a series of benzylidene ketone-based twophoton initiators containing double bonds and dialkylamino groups, synthesized through one-step aldol condensation reactions ${ }^{1,10}$. Compared to other types, these initiators offer $D-\pi-A-\pi-D$ core structures with simplicity in synthesis and reduced cost ${ }^{1}$. Meanwhile, initiators with multi-branched structures have been observed to offer a cooperative enhancement of TPA in these structures $10,25,26,27$. These developments offer the potential for easily manufacturable PIs that can increase the processing range for TPIP and increase the fabrication speed, often seen as a bottleneck in the TPIP based process.

In this paper, an extension to established benzylidene ketone-based initiators will be shown, demonstrating that the addition of multiple branches can be beneficial for TPIP ${ }^{26,28}$, including higher initiation efficiency and faster fabrication speed. The synthesis of initiators and preparation of resins will be described, before characterisation of TPIP structures is presented, with a direct comparison to results using a commercially available PI offered (Irgacure 369). These new materials can be useful add-ons to be used in the development of TPIP monomers and increase the materials palette for TPIP.

\section{METHODOLOGY}

\subsection{Materials:}

4-(Dimethylamino)benzaldehyde, cyclohexanone, 1,3-cyclohexanedione, 1,4-cyclohexanedione, ethanol, sodium hydroxide and ammonium chloride were purchased from Sigma-Aldrich UK and used as received. 2-Benzyl-2-(dimethylamino)-4'-morpholinobutyrophenone (Irgacure 369) was purchased from Sigma-Aldrich and served as a reference photoinitiator ${ }^{28}$. Pentaerythritol triacrylate (PETA) (Sigma-Aldrich) was used as the monomer for polymer resins. The synthetic routes for the final compounds (a) 2,6-Bis(4-(dimethylamino)benzylidene)cyclohexanone (2-BI), (b) 4,6-bis(4(dimethylamino)benzylidene)-2-(4-(dimethylamino)benzylidene)cyclohexane-1,3-dione (3-BI) and (c) 2,3,5,6-tetrakis(4-(dimethylamino)benzylidene)cyclohexane-1,4-dione (4-BI) are shown in Scheme 1 with their details given in section 2.2 . 


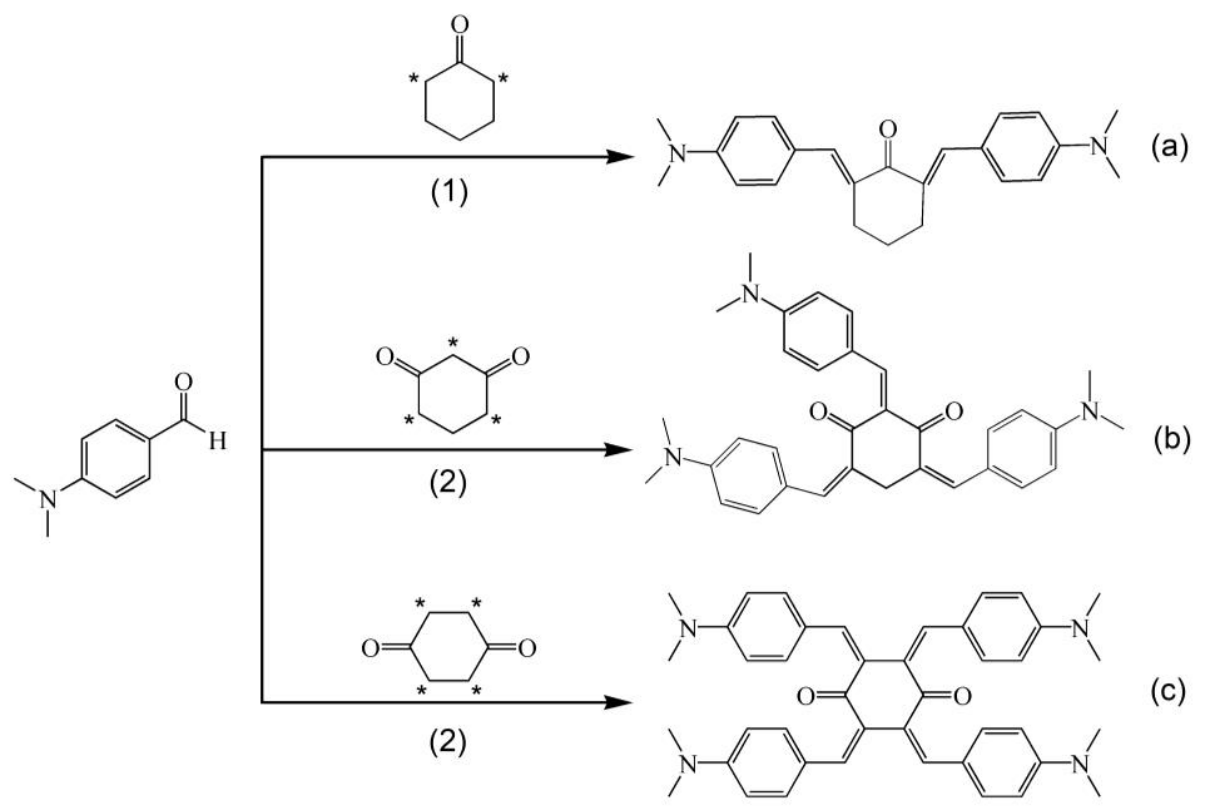

Scheme 1. Reaction Scheme for the Synthesis of (a) 2-branched two-photon initiators 2-BI, (b) 3branched two-photon initiator 3-BI, and (c) 4-branched two-photon initiator 4-BI

\subsection{Synthesis of initiators}

Two, three and four-branched initiators 2-BI, 3-BI and 4-BI were synthesised using a method similar to that reported by Li et $\mathrm{al}^{1}$. 20, 30, and $40 \mathrm{mmol}$ of benzaldehyde and $10 \mathrm{mmol}$ of cycloketone, 1,3cyclohexanedione, and 1,4-cyclohexanedione respectively were mixed with $40 \mathrm{~mL}$ of ethanol in a $100 \mathrm{ml}$ round bottom flask. $10 \mathrm{mmol}$ of sodium hydroxide was dissolved in $3 \mathrm{ml}$ of deionized water, and the solution was added dropwise into the vigorously stirred mixture. After stirring for 12 hours, the mixture was diluted with $200 \mathrm{~mL}$ of chloroform and washed using saturated $\mathrm{NH}_{4} \mathrm{Cl}$ three times to remove the $\mathrm{NaOH}$. The organic phase was collected using a separating funnel, followed by a solvent evaporation process. The products were purified by recrystallization from ethanol. 2-BI ${ }^{1} \mathrm{H}$ NMR (400 $\mathrm{MHz}, \mathrm{CDCl} 3) \delta 7.79(\mathrm{~s}, 2 \mathrm{H}), 7.47(\mathrm{~d}, \mathrm{~J}=8.9 \mathrm{~Hz}, 4 \mathrm{H}), 6.75(\mathrm{t}, \mathrm{J}=5.9 \mathrm{~Hz}, 4 \mathrm{H}), 3.05(\mathrm{~s}, 12 \mathrm{H}), 3.03-2.92$ $(\mathrm{m}, 4 \mathrm{H}), 1.84(\mathrm{t}, \mathrm{J}=6.2 \mathrm{~Hz}, 2 \mathrm{H}) .3-\mathrm{BI}{ }^{1} \mathrm{H}$ NMR $(400 \mathrm{MHz}$, DMSO) $\delta 9.68(\mathrm{~s}, 1 \mathrm{H}), 7.70(\mathrm{~d}, \mathrm{~J}=9.0 \mathrm{~Hz}, 2 \mathrm{H})$, $7.48-7.37(\mathrm{~m}, 1 \mathrm{H}), 7.20(\mathrm{~m}, \mathrm{~J}=8.6,1.7 \mathrm{~Hz}, 4 \mathrm{H}), 7.03-6.95(\mathrm{~m}, 1 \mathrm{H}), 6.81(\mathrm{~d}, \mathrm{~J}=8.9 \mathrm{~Hz}, 2 \mathrm{H}), 6.77-$ $6.59(\mathrm{~m}, 6 \mathrm{H}), 4.84(\mathrm{~s}, 2 \mathrm{H}), 4.41(\mathrm{~s}, 1 \mathrm{H}), 3.06(\mathrm{~s}, 6 \mathrm{H}), 2.96(\mathrm{~s}, 6 \mathrm{H}), 2.88(\mathrm{~s}, 12 \mathrm{H}), 1.68-1.63(\mathrm{~m}, 1 \mathrm{H}) .4-$ $\mathrm{BI}{ }^{1} \mathrm{H}$ NMR (400 MHz, DMSO) $\delta 9.68(\mathrm{~s}, 3 \mathrm{H}), 8.33(\mathrm{~s}, 2 \mathrm{H}), 7.97(\mathrm{~s}, 1 \mathrm{H}), 7.73-7.60(\mathrm{~m}, 8 \mathrm{H}), 6.80(\mathrm{~d}, \mathrm{~J}=$ $8.9 \mathrm{~Hz}, 8 \mathrm{H}), 3.33(\mathrm{~s}, 12 \mathrm{H}), 3.06(\mathrm{~s}, 12 \mathrm{H}), 2.10(\mathrm{~s}, 2 \mathrm{H})$.

The ${ }^{1} \mathrm{H}$ NMR spectra of 3-BI and 4-BI show the presence of the products and some traces of the initial reactant benzaldehyde. These products are difficult to be fully purified from the reactants due to the similarity in polarity and future efforts will be required to purify these products, for example using a chromatograph. The ${ }^{1} \mathrm{H}-\mathrm{NMR}$ spectra of the synthesized initiators are included in the supporting materials. 


\subsection{Resin preparation}

Pentaerythritol triacrylate (PETA) is a commonly employed monomer for TPIP and as consequence was chosen for use in this study due to its reliability ${ }^{10,12,19,29}$. For each resin, $0.02 \mathrm{~g}$ of each initiator was dissolved in $0.20 \mathrm{~g}$ of dichloromethane, followed by dispersal in $2.00 \mathrm{~g}$ of PETA. The mixtures were fully mixed using a magnetic stirrer at $600 \mathrm{rpm}$ for 30 minutes, followed by ultrasonication for a further 30 minutes. The structures for each of the molecules are shown in Figure 1.

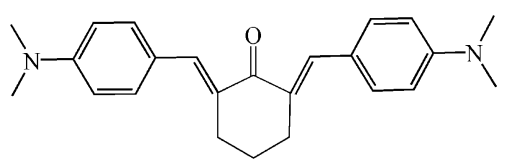

(a)<smiles>CCC(Cc1ccccc1)(C(=O)c1ccc(N2CCOCC2)cc1)N(C)C</smiles>

(d)

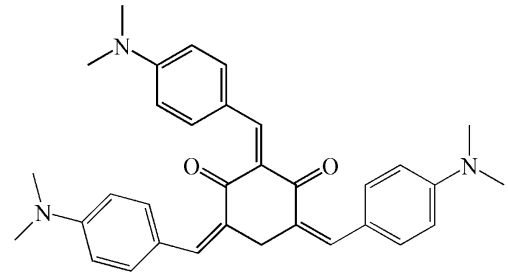

(b)

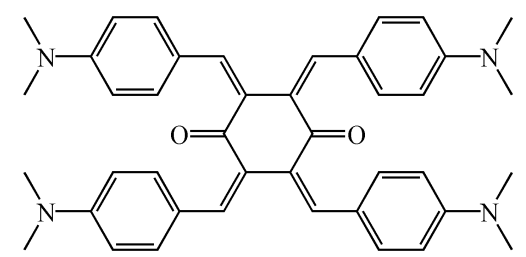

(c)<smiles>C=CC(=O)OCC(CO)(COC(=O)C=C)COC(=O)C=C</smiles>

(e)

Figure 1. Chemical structures of synthesised two-photon initiators (a) 2-BI, (b) 3-BI, (c) 4-BI, (d) reference initiator Irgacure 369 (1369) and (e) the monomer PETA

\subsection{TPIP Structuring Tests}

Two-photon polymerisation: A Nanoscribe Photonic Professional GT two-photon lithography system was used to initiate the polymerisation of the resins. The system uses a laser beam at a wavelength of $780 \mathrm{~nm}$, pulse frequency of $80 \mathrm{MHz}$ and pulse duration of $120 \mathrm{fs}$, which was focused by an oil immersion objective (with a magnification of 63x, numerical aperture (NA) of 1.4 and working distance (WD) of $190 \mu \mathrm{m}$ ).

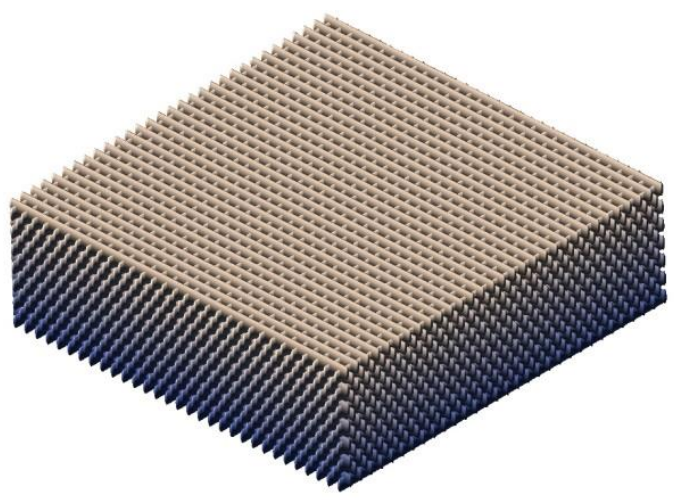

(a)

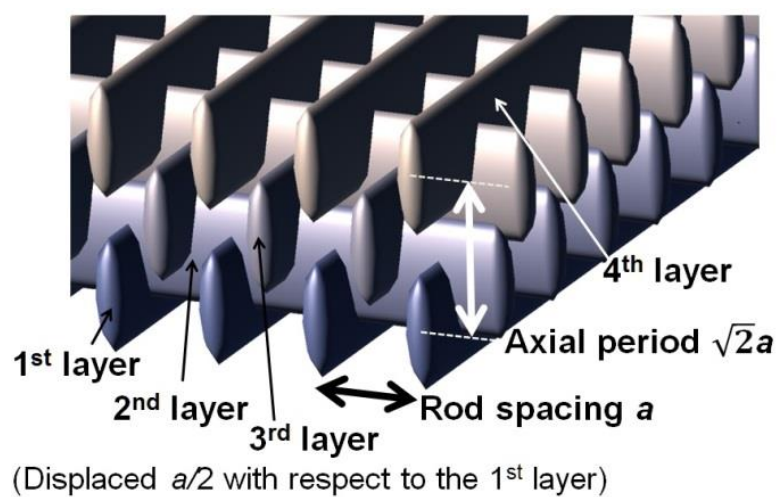

(b)

Figure 2. (a) Illustration of the woodpile structure with FCC lattice; (b) Illustration of the FCC lattice with a rod spacing $a$ and a lattice period of $\sqrt{2} a$ 
General Procedure: For all samples the same fabrication process was implemented: The optical material was deposited onto a glass substrate which was then loaded into the Nanoscribe for twophoton laser writing to produce a 3D structure inside the material volume. After laser writing, the unpolymerised monomer was removed by developing the structure in propylene glycol monomethyl ether acetate (PGMEA) and subsequently in 2-propanol. Face-centred-cubic (FCC) woodpile structures $^{30,31}$ (with a rod spacing $a=1 \mu \mathrm{m}$, as illustrated in Figure 1) were fabricated under laser powers ranging from $15 \mathrm{~mW}$ to $50 \mathrm{~mW}$ and writing speeds ranging from $5 \mathrm{~K} \mu \mathrm{m} / \mathrm{s}$ to $100 \mathrm{~K} \mu \mathrm{m} / \mathrm{s}$ for the evaluation. The resulting structures, including the structural dimensions and integrity, were studied using a Hitachi TM3000 Tabletop Scanning Electron Microscope (SEM) and a Hitachi S-2600N SEM. A Nikon Reichert-Jung MEF3 optical microscope was used to characterise the quality of the printed structures.

\section{RESULTS AND DISCUSSIONS}

\subsection{TPIP processing windows}

(a)

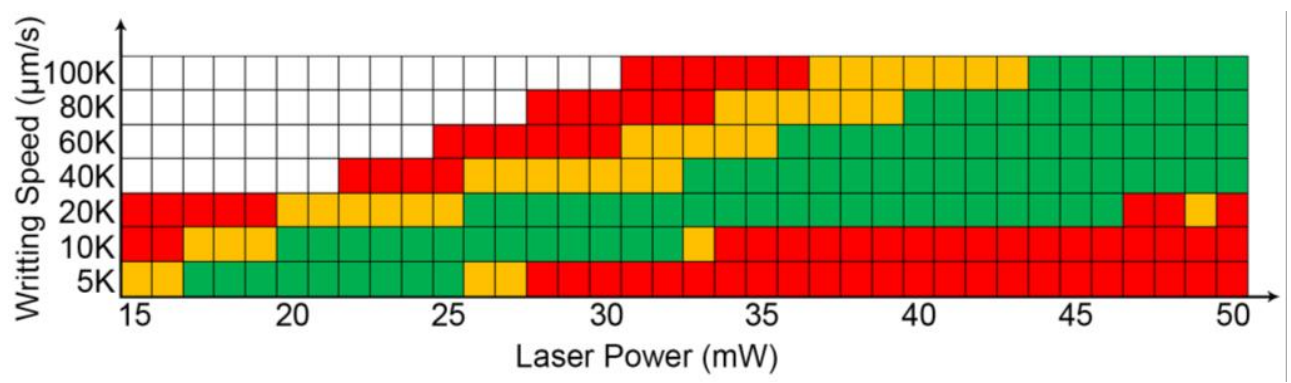

(b)

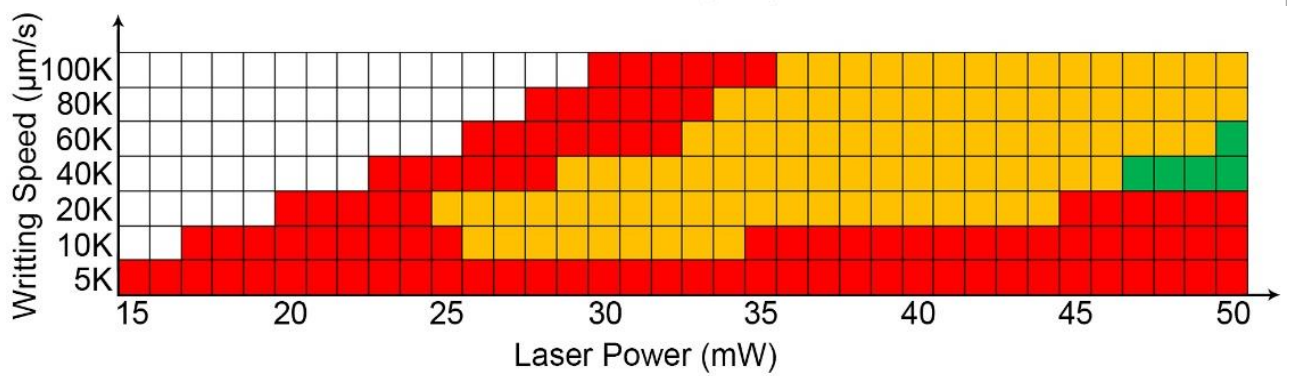

(c)

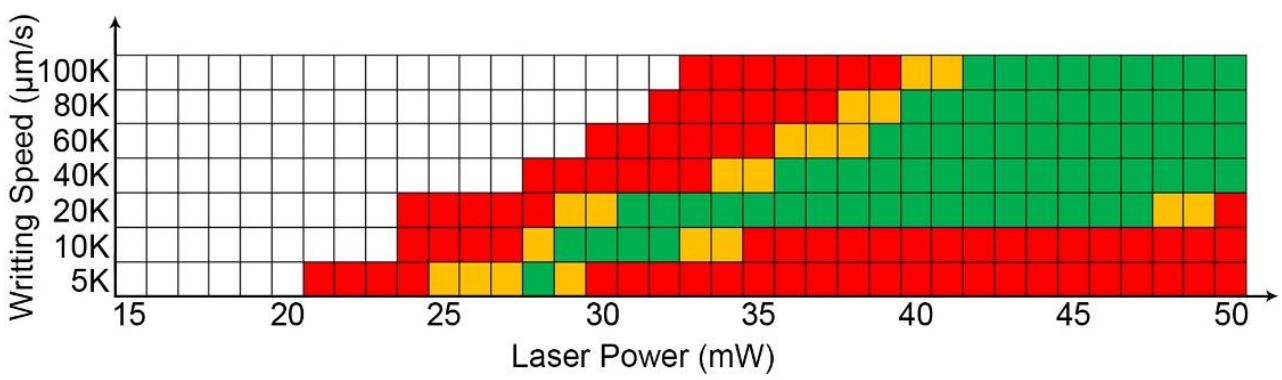

(d)

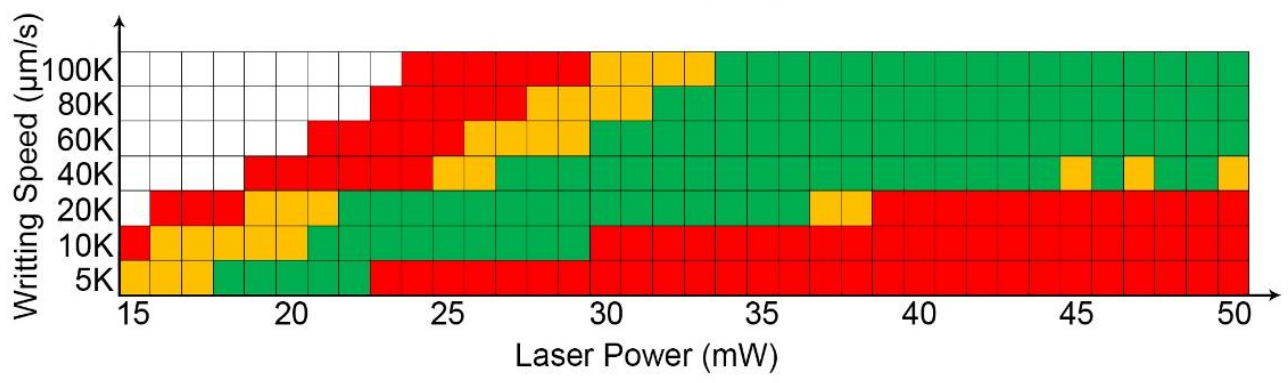


(e)

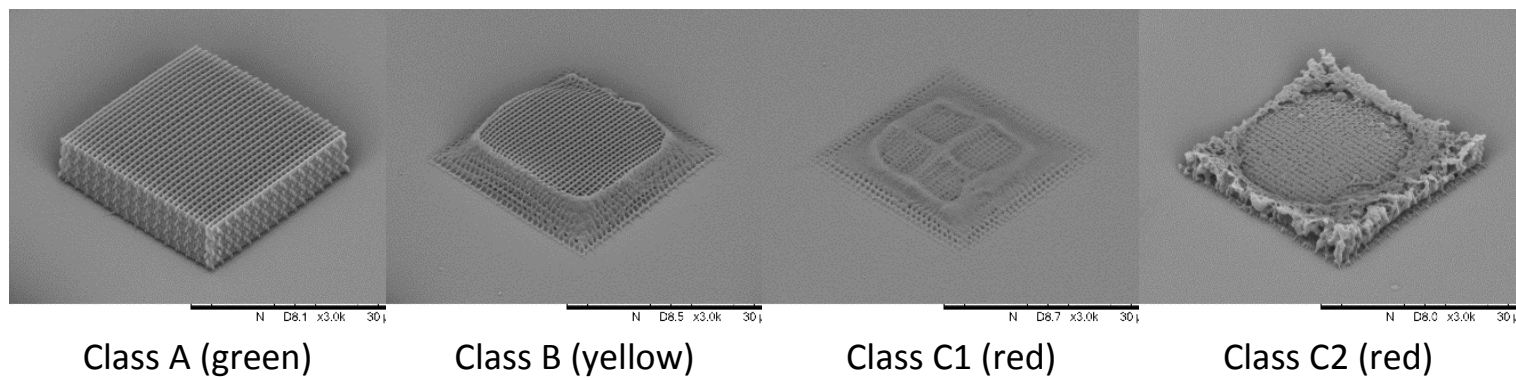

Figure 3. Processing windows in TPIP screening tests performed with different speeds and laser power using all four resins (a) PETA/I369, (b) PETA/2-BI, (c) PETA/3-BI and (d) PETA/4-BI. The qualities of structures were classified and marked using different colours. (e) Classification of the structures by the typical quality of their appearances. Green - Class A; Yellow - Class B; Red - Class C; white-Class D.

Having synthesized the new compounds, a series of experiments were performed to compare the efficiencies of the initiators by fabrication of complex 3D structures with respect to write speed and laser power. Both limits were sought according to the qualities of the resulting structures, since one would like to increase the fabrication speed through increase of power and/or increase of write speed $^{28}$, but an increased laser power may also have the negative effect of degenerating the structure $^{28}$. To allow for rapid comparison independent of the structure, arrays of woodpile structures were fabricated (as shown in Figure 2) using an increasing selection of laser power and writing speed (Figure 3). The laser power was varied from $15 \mathrm{~mW}$ to $50 \mathrm{~mW}$ in increments of $1 \mathrm{~mW}$, whilst writing speeds of $5 \mathrm{~K}, 10 \mathrm{~K}, 20 \mathrm{~K}, 40 \mathrm{~K}, 60 \mathrm{~K}, 80 \mathrm{~K}$ and $100 \mathrm{~K} \mu \mathrm{m} / \mathrm{s}$ were chosen.

Qualities of the woodpile structures are greatly dependent on the processing range of TPIP (a combination of laser power and writing speeds), exceeding the ideal range can result in malforming of objects. When subjected to closer scrutiny a number of phenomena were identified. When the laser intensity was too low, an insufficient number of free radicals were generated and hence insufficient polymerisation occurred in the exposed area, causing the fabricated structures to be weak and inaccurate. If the laser intensity was too high, it would generate excessive heat within the exposed area of resin, causing bubbles or even polymer decomposition ${ }^{28}$.

As can be seen from the processing windows shown in Figure 3, the different colour of the grids and their corresponding classes were used to illustrate the evaluated TPA initiation efficiency of the initiators. Four classes were employed to indicate the quality of the structures (Figure $3 e$ ). Class A (green) defines excellent structures with fine hatch lines and sharp edges, while class B (yellow) indicates contorted structures with some shape changes, as well as other small mistakes such as holes and slightly deformed edges. Structures rated as class C (red) are no longer identifiable, which can be either due to insufficient polymerisation (Class $\mathrm{C} 1$ ) or polymer decomposition caused by overexposure (Class C2). No structure is observed for class D (white), indicating insufficient laser intensities for polymerisation to happen.

The commercially available initiator $1369^{28}$ allowed the fabrication of excellent woodpile structures at suitable laser power and writing speeds. For a writing speed of $5 \mathrm{~K} \mu \mathrm{m} / \mathrm{s}$, laser powers between 17 $\mathrm{mW}$ and $25 \mathrm{~mW}$ resulted in fine structures. As the writing speed increased from $5 \mathrm{~K}$ to $100 \mathrm{~K} \mu \mathrm{m} / \mathrm{s}$, 
the resin required laser power higher than $44 \mathrm{~mW}$ to be cured, as one would expect from a consideration of the energy density incident during the process. This can be described using the following simple relation (1):

$$
\rho_{E}=\frac{v}{k} \frac{d}{P_{0}}
$$

where $\rho_{E}$ is the energy density, $v$ is the write speed, $k$ is the ratio of the laser power to the maximum laser power, $P_{0}$, and $d$ is the laser spot diameter. If we consider that the energy density required for polymerisation will remain constant for a given system, then any increase in the writing speed must be matched by an increase in laser power, or vice versa. This also gives a qualitative measure of the 'efficiency' of the process, since if the gradient of the border between green and yellow (class $A$ and class $B$ ) increases (i.e., $v / k$ ), then so must the effective energy density, i.e., the number of photons absorbed and promoting polymerisation must be increasing, pushing the range of processing into apparently lower ranges of energy density.

For all 252 combinations of laser power and writing speed shown in the processing windows in Figure 3, 94 of them achieved class A structures using initiator 1369, indicating a large ideal processing range for TPIP. 2-BI showed a very poor result, only 5 out of 252 achieved class A. For 3-BI and 4-BI, this value increased to 69 and 107, respectively. Although at first sight of Figure 3, 3-BI and 4-BI did not show significant improvement in the ideal processing range, they showed improvements at high writing speed of $100 \mathrm{~K} \mu \mathrm{m} / \mathrm{s}$, particularly in the case of 4-BI. In this case higher maximum write speeds, lower laser powers and a steepening of the border between yellow and green regions in Figure 3(d) all point to a more effective and potentially more efficient PI that can enable faster processing. For synthesised initiators $2-\mathrm{BI}, 3-\mathrm{BI}$ and $4-\mathrm{BI}$, since they all have dimethylamino groups as electron-donors, vinyl as $\pi$-conjugated bridges, and carbonyl as electron acceptor groups, it can be hypothesised that the larger number of branches leads to higher donor-to-acceptor ratios and an improved efficiency.

\subsection{Energy thresholds for initiator 4-BI}

The increase in 4-BI performance in comparison to 1369 suggests that this is a promising highefficiency two-photon initiator that could enable the fabrication of the complex 3D structures that are being sought in AM. To determine the quality of the resin PETA/4-BI, the thresholds for polymerisation and decomposition were investigated by fabricating lines using single sweeps at various writing speeds and laser powers ${ }^{32}$ and then measuring the line widths from SEM images (Figure 4b). One example is shown in Figure 4a, an array of lines were fabricated with a fixed writing speed of $10 \mathrm{~K} \mu \mathrm{m} / \mathrm{s}$ and varying laser power from $0.5 \mathrm{~mW}$ to $50 \mathrm{~mW}$. To avoid issues with interface errors, each of the 5 vertical line repeats were fabricated on top of a series of horizontal lines printed beforehand using the same resin with a fixed laser power and writing speed; these acted as supporting structures and ensured a level base on which to build. Figure $4 \mathrm{~b}$ shows that lower writing speeds and higher laser power resulted in larger line width owing to the larger energy density and an increase in the area over which the intensity of photon incidence is in excess of the threshold required for initiation of polymerisation. For a successful TPIP process, the energy density must sit within the range bounded at the upper end of polymer decomposition and at the lower end incomplete polymerisation. 

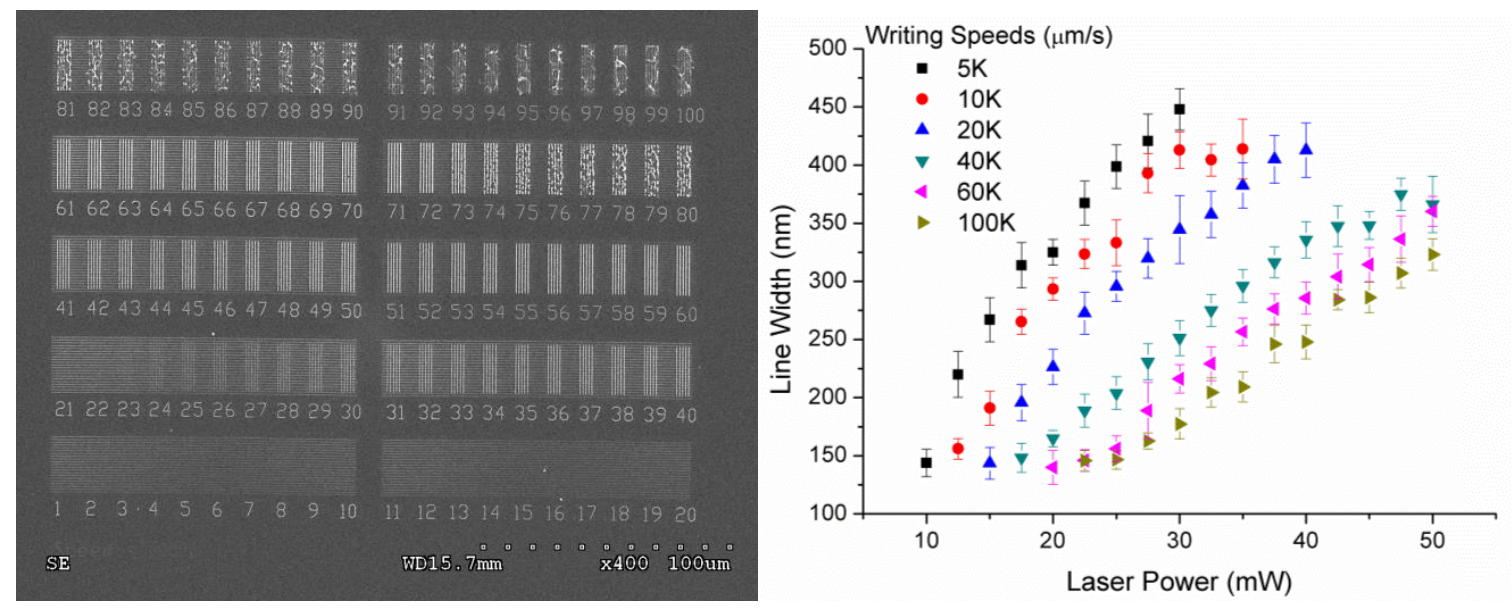

Figure 4. (a) SEM image showing an array of fabricated lines drawn using single laser sweeps with a writing speed of $10 \mathrm{~K} \mu \mathrm{m} / \mathrm{s}$ and varying laser power percentage $(100 \%$ corresponding to a laser power of $50 \mathrm{~mW}$ ); (b) and their corresponding widths in $\mathrm{nm}$.

The thresholds for polymerisation and decomposition of resin PETA/4-BI were determined by observing the integrity of the lines drawn using different laser powers (Figure 5(a)). For samples printed at $10 \mathrm{~K} \mu \mathrm{m} / \mathrm{s}$ writing speed, the threshold for polymerisation was determined to be $11.5 \mathrm{~mW}$, while the threshold for decomposition was $36 \mathrm{~mW}$. Figure 5(b) illustrates the ideal laser power range for the resin PETA/4-BI. As can be seen from the results, the laser power $s$ increased with writing speed. For writing speeds higher than $40 \mathrm{~K} \mu \mathrm{m} / \mathrm{s}$, the threshold for decomposition was beyond the maximum laser power tested in current study which was $50 \mathrm{~mW}$. This range is slightly wider than the results in figure 3 , which was due to the complex woodpile structures being more likely to suffer from local heat accumulation than simple lines, causing a narrower laser power range.
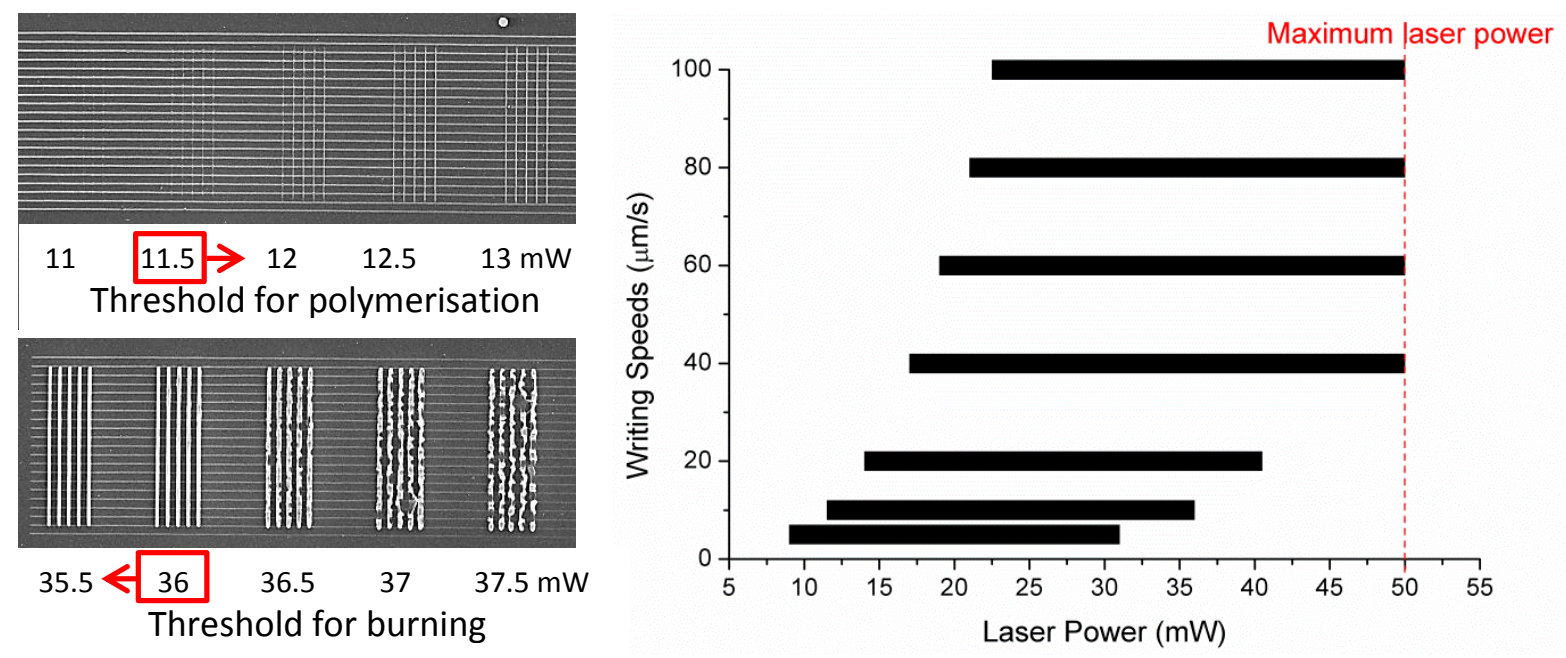

Figure 5 (a) Thresholds for polymerisation and decomposition were defined according to the integrity of the lines; (b) the laser power thresholds for polymerisation and decomposition under different writing speeds.

By selecting suitable combinations of writing speeds and laser power, complex 3D structures, including bracelet (figure 6a), University logo (figure 6b) and hollowed pyramid (figure 6c) were fabricated, demonstrating the potential for PETA/4-BI as a TPIP/3D printing formulation; a promising 
development since such micron/nano scale suspending 3D structures are not achievable using other techniques such as inkjet printing and traditional photolithography.

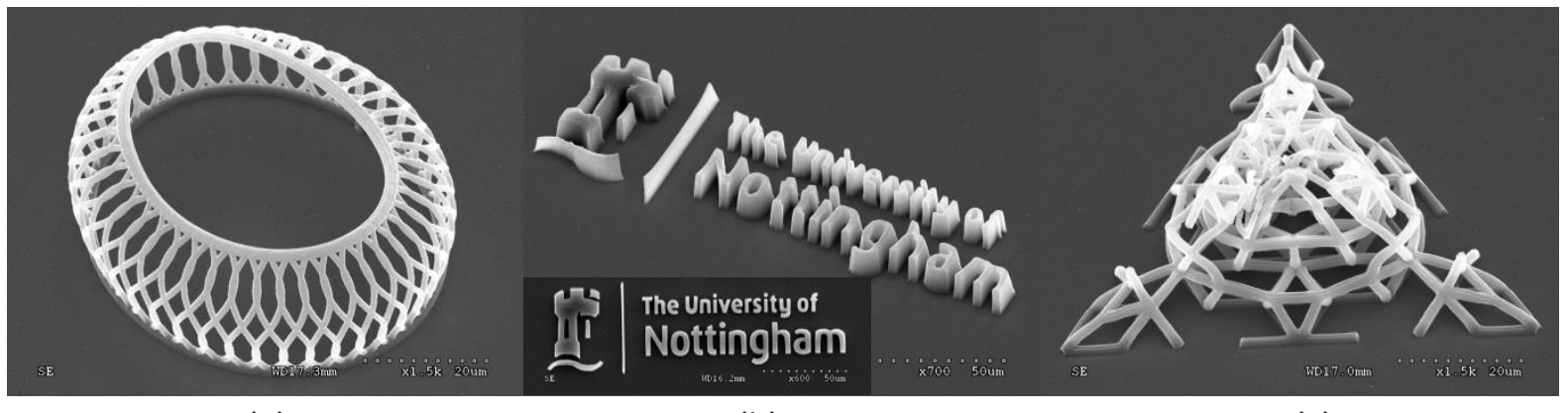

(a)

(b)

(c)

Figure 6. SEM images of complex 3D structures fabricated using resin PETA/4-BI with optimized parameters. Samples were tilted at $45^{\circ}$. The insert image in (b) was taken without tilting.

\section{CONCLUSIONS}

In this work, a series of benzylidene ketone-based two-photon initiators with multi-branched structures were synthesised and used as TPA initiators. The TPIP processing window results indicate that increasing the number of branches on the molecules resulted in a larger ideal processing window. At a laser beam wavelength of $780 \mathrm{~nm}$ and a photo-initiator concentration of $1 \mathrm{wt} . \%$, the four-branched initiator 4-BI exhibited excellent performance in the TPIP tests with both higher writing speeds and broader ideal processing windows compared to commercial initiator Irgacure 369. At a writing speed of $100 \mathrm{~K} \mu \mathrm{m} / \mathrm{s}, 4-\mathrm{BI}$ required a much lower laser power of $34 \mathrm{~mW}$ to fabricate 3D woodpile structures compared with the $44 \mathrm{~mW}$ of Irgacure 369. Its detailed laser intensity range was studied by fabrication of simple lines and complex 3D structures. These results indicate that these new multi-branched initiators could potentially increase the fabrication efficiency and have extensive application prospects in a wide range of AM processes, e.g., micro-fabrication and high density optical data storage.

\section{ACKNOWLEDGMENTS}

The authors would like to acknowledge funding support fromUniversity of Nottingham, the EPSRC (Grant number EP/1033335/2).

\section{REFERENCES}

1. Li, Z. et al. A straightforward synthesis and structure-activity relationship of highly efficient initiators for two-photon polymerization. Macromolecules 46, 352-361 (2013).

2. He, Y., Wildman, R. D., Tuck, C. J., Christie, S. D. R. \& Edmondson, S. An Investigation of the Behavior of Solvent based Polycaprolactone ink for Material Jetting. Nat. Publ. Gr. 1-10 (2016). doi:10.1038/srep20852

3. Gunasekera, D. H. A. T. et al. Three dimensional ink-jet printing of biomaterials using ionic liquids and co-solvents. Faraday Discuss. (2016). doi:10.1039/C5FD00219B

4. Zhou, C. \& Chen, Y. Additive manufacturing based on optimized mask video projection for improved accuracy and resolution. J. Manuf. Process. 14, 107-118 (2012). 
5. Hart, L. R. et al. 3D Printing of Biocompatible Supramolecular Polymers and their Composites.ACS Appl. Mater. Interfaces. (2016). doi:10.1021/acsami.5b10471

6. Sun, Y. Y. et al. Solvent inkjet printing process for the fabrication of polymer solar cells. Rsc Adv. 3, 11925-11934 (2013).

7. Zhang, F. et al. Inkjet printing of polyimide insulators for the 3D printing of dielectric materials for microelectronic applications. J. Appl. Polym.Sci. 43361, 1-11 (2016).

8. Hu, Q., Tuck, C., Wildman, R. \& Hague, R. in Handbook of Nanoparticles (ed. Aliofkhazraei, M.) 1219-1278 (Springer International Publishing, 2016). doi:10.1007/978-3-319-15338-4_55

9. Conner, B. P. et al. Making sense of 3-D printing : Creating a map of additive manufacturing products and services. Addit. Manuf. 1-4, 64-76 (2014).

10. Wu, J. et al. Multibranched benzylidene cyclopentanone dyes with large two-photon absorption cross-sections. New J. Chem. 30, 1098 (2006).

11. Li, Z. et al. Initiation efficiency and cytotoxicity of novel water-soluble two-photon photoinitiators for direct 3D microfabrication of hydrogels. RSC Adv. 3, 15939 (2013).

12. Li, Z. et al. Synthesis and structure-activity relationship of several aromatic ketone-based twophoton initiators. J. Polym. Sci. Part A Polym. Chem. 49, 3688-3699 (2011).

13. Maruo, S., Nakamura, O. \& Kawata, S. Three-dimensional microfabrication with two-photonabsorbed photopolymerization. Opt. Lett. 22, 132-134 (1997).

14. Malval, J. P. et al. Enhancement of the two-photon initiating efficiency of a thioxanthone derivative through a chevron-shaped architecture. Chem. Mater. 23, 3411-3420 (2011).

15. Zhou, W. et al. An efficient two-photon-generated photoacid applied to positive-tone 3D microfabrication. Science 296, 1106-1109 (2002).

16. Denk, W. Two-photon scanning photochemical microscopy: mapping ligand-gated ion channel distributions. Proc. Natl. Acad. Sci. U. S. A. 91, 6629-6633 (1994).

17. Williams, R. M., Piston, D. W. \& Webb, W. W. Two-photon molecular excitation provides intrinsic 3-dimensional resolution for laser-based microscopy and microphotochemistry. FASEB J. 8, 804-813 (1994).

18. Parthenopoulos, D. A. \& Rentzepis, P. M. Three-Dimensional Optical Storage Memory. Science (80-. ). 245, 843-845 (1989).

19. Gan, Z., Cao, Y., Evans, R. a \& Gu, M. Three-dimensional deep sub-diffraction optical beam lithography with $9 \mathrm{~nm}$ feature size. Nat. Commun. 4, 2061 (2013).

20. Xing, J. et al. A water soluble initiator prepared through host-guest chemical interaction for microfabrication of 3D hydrogels via two-photon polymerization. J. Mater. Chem. B 2, 43184323 (2014).

21. Xing, J.-F. et al. C2v symmetrical two-photon polymerization initiators with anthracene core: synthesis, optical and initiating properties. Phys. Chem. Chem. Phys. 14, 15785-92 (2012).

22. Schafer, K. J. et al. Two-photon absorption cross-sections of common photoinitiators. J. Photochem. Photobiol. A Chem. 162, 497-502 (2004).

23. He, G. S., Tan, L. S., Zheng, Q. \& Prasad, P. N. Multiphoton absorbing materials: Molecular designs, characterizations, and applications. Chem. Rev. 108, 1245-1330 (2008).

24. Xue, J., Zhao, Y., Wu, F. \& Fang, D. C. Effect of bridging position on the two-photon polymerization initiating efficiencies of novel coumarin/benzylidene cyclopentanone dyes. J. Phys. Chem. A 114, 5171-5179 (2010).

25. Chung, S.-J. et al. Cooperative Enhancement of Two-Photon Absorption in Multi-branched Structures. J. Phys. Chem. B 103, 10741-10745 (1999).

26. Zhao, Y., Li, X., Wu, F. \& Fang, X. Novel multi-branched two-photon polymerization initiators of ketocoumarin derivatives. J. Photochem. Photobiol. A Chem. 177, 12-16 (2006).

27. Yan, Y. et al. A new multi-branched two-photon photopolymerization initiator: Synthesis and non-linear optical properties of a 1,3,5-triazine-based octupolar molecule. Mater. Chem. Phys. 90, 139-143 (2005). 
28. Passinger, S. Two-Photon Polymerization and application to Surface Plasmon Polaritons. (Cuvillier-Verlag Gottingen, 2008). Doi: 978-3-86727-662-7

29. Xue, J., Zhao, Y., Wu, J. \& Wu, F. Novel benzylidene cyclopentanone dyes for two-photon photopolymerization. J. Photochem. Photobiol. A Chem. 195, 261-266 (2008).

30. Taverne, M. P. C., Ho, Y. D. \& Rarity, J. G. Investigation of defect cavities formed in threedimensional woodpile photonic crystals. J. Opt. Soc. Am. B 32, 639 (2015).

31. Fischer, J. \& Wegener, M. Three-dimensional direct laser writing inspired by stimulatedemission-depletion microscopy. Opt. Mater. Express 1, 2363-2366 (2011).

32. Xing, J.-F. et al. Improving spatial resolution of two-photon microfabrication by using photoinitiator with high initiating efficiency. Appl. Phys. Lett. 90, 131106-131106-3 (2007). 